\title{
Evolution of a Percutaneous Fetoscopic Access System for Single-Port Tracheal Occlusion
}

\author{
By Constantinos A. Hajivassiliou, Scott M. Nelson, Peta D. Dunkley, Alan D. Cameron, \\ Tim G. Frank, Alfred Cuschieri, and Graham Haddock \\ Glasgow, Scotland and Dundee, Scotland
}

\begin{abstract}
Background/Purpose: Prenatal tracheal occlusion currently is being assessed as a treatment modality for congenital diaphragmatic hernia (CDH). The development of a totally percutaneous fetoscopic access system would help avoid the need for maternal laparotomy and reduce the morbidity rate of fetal surgical procedures for the mother. Laparoscopic radial expansion sheaths and Seldinger technique-based vascular catheters both have been advocated as means of achieving amniotic cavity access. The authors have investigated these 2 systems in an attempt to develop a reliable method for achieving safe percutaneous fetoscopic access and present the first successful attempt to deploy an intratracheal balloon using an entirely percutaneous approach through a single port in an ovine model.
\end{abstract}

Methods: A number of prototype systems were evaluated sequentially over a 3 -year period in an ovine model: (1) the radially expanding InnerDyne step port system, (2) a new rigid cannula with a bulbous/sharp end preloaded onto the radially expanding InnerDyne port, (3) a conical removable addition to the rigid cannula in 2, (4) a modified bulbous/ sharp ended cannula incorporating a circumferential protective insert, (5) a rigid split sheath with the radially expanding port placed through the lumen of the split sheath, (6) a flexible introducer and dilator with the split sheath (used in the Seldinger placement of central lines), and (7) a 2-needle approach using a superelastic shape-memory alloy NickelTitanium wire with the flexible dilator and sheath, incorporating a side perfusion port. For balloon tracheal occlusion, live anaesthetized time-mated pregnant ewes were used at 110 days' gestation. Tracheobronchoscopy was achieved using a $3-\mathrm{mm}^{\circ}$ telescope, and the cutaneotracheal tract was secured by a $3.3-\mathrm{mm}$ sheath incorporating a side-perfusion port. The rigid telescope was replaced by a flexible chole- dochoscope preloaded with a silicone balloon. The balloon was deployed $2 \mathrm{~cm}$ above the carina proximal to the right upper lobe bronchus.

Results: The many problems encountered in the evolution of the preferred system related mainly to separation and tenting of the chorioamniotic membranes in the ovine uterus and inconsistent access to the fetal parts of interest. Each resulted in significant modifications to our approach. Furthermore, the use of rigid access devices commonly caused fetal injury. Successful access to the intrauterine cavity and cannulation of the trachea was achieved consistently with minimal trauma, irrespective of fetal position by method 7 . Multiple port placement allowed visualization of the entry of all components of the system confirming minimal chorioamniotic membrane separation and tenting. Single port tracheal occlusion was undertaken first on 6 cadavers before being performed successfully on 3 live anaesthetized ewes. Fetoscopic access and cannulation of the trachea was achieved consistently in all live animals irrespective of fetal position.

Conclusions: This modified Seldinger technique using the unique properties of the memory-shaped alloy wire for initial uterine access offers a safe method for the percutaneous placement of fetoscopic ports in the ovine model for prenatal intervention. Successful placement of a tracheal balloon entirely through a single percutaneously placed port represents a further advance in prenatal therapy for $\mathrm{CDH}$.

J Pediatr Surg 38:45-50. Copyright 2003, Elsevier Science (USA). All rights reserved.

INDEX WORDS: Congenital diaphragmatic hernia, pulmonary hypoplasia, fetal surgery, fetoscopy, tracheal occlusion, prenatal, fetus, lung, ultrasound, endoscopy, sheep.
$\mathbf{E}$ NDOSCOPIC surgical technology has developed to such a degree that it now offers the opportunity to develop techniques that may be adapted for prenatal fetal intervention. ${ }^{1}$ To date, fetal endoscopy has been achieved, both in animal models and in humans, by laparotomy with transmyometrial port placement, ${ }^{2}$ which is associated with significant maternal morbidity. The use of a percutaneous fetoscopic access system with associated decreased maternal morbidity, minimal uterine instrumentation, small chorioamniotic membrane defects, and decreased nontraumatic fetal distress, may enable a reduction in the incidence of premature labour.

Radially expanding laparoscopic ports have been developed to allow for flexibility of port size and a reduced
From the Department of Paediatric Surgery, Royal Hospital for Sick Children and University of Glasgow; the Department of Obstetrics and Fetal Medicine, Queen Mother's Hospital, Glasgow, Scotland; and Tayside Institute of Child Health and Department of Surgery and Molecular Oncology, Ninewells Hospital \& Medical School, Dundee, Scotland.

Presented at the 49th Annual Congress of the British Association of Paediatric Surgeons, Cambridge, England, July 23-26, 2002.

This study was supported by the Wellcome Trust and the Scottish Hospital Endowment Research Trust.

Address reprint requests to Constantinos A. Hajivassiliou, Consultant Paediatric/Neonatal Surgeon, Department of Surgical Paediatrics, Royal Hospital for Sick Children, Yorkhill, Glasgow, G3 8SJ, Scotland. Copyright 2003, Elsevier Science (USA). All rights reserved. 0022-3468/03/3801-0008\$35.00/0

doi:10.1053/jpsu.2003.50007 


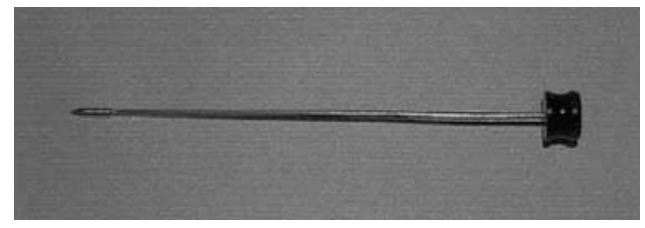

Fig 1. Prototype rigid introducer to deploy innerdyne radially expandable port.

incidence of trochar site injury. ${ }^{3}$ Similarly, placement of large intravascular catheters using the Seldinger technique also is associated with minimal tract trauma. The ovine model has been used widely as a system for developing fetal interventions. ${ }^{4,5}$ However, the anatomy of the chorioamniotic membranes in the ewe, which are not closely adherent to the uterine wall, ${ }^{6}$ present a significant challenge to percutaneous puncture and port passage. We have investigated 7 access systems in the ovine model and examined the feasibility of deploying an intratracheal balloon using an entirely percutaneous approach through a single port as a potential method for prenatal therapy of $\mathrm{CDH}$.

\section{MATERIALS AND METHODS}

The Animal Ethics Committee of the University of Glasgow Veterinary School oversaw all animal work. The United Kingdom Home Office approved all procedures under the terms of the current UK animal legislation.

Port development was undertaken on cadaveric ewes and live anaesthetized time-mated pregnant Texel Cross ewes at 110 days' gestation. Anaesthetised ewes were premedicated with $0.2 \mathrm{mg} / \mathrm{kg}$ diazepam (Diazepam Injection BP; Phoenix Pharma, Gloucester, UK). Anaesthesia was induced intravenously with $9 \mathrm{mg} / \mathrm{kg}$ Saffan (Saffan; ScheringPlough Animal Health, Welwyn Garden City, UK) and maintained with $70 \%$ nitrous oxide, $27.5 \%$ oxygen, $2.5 \%$ isoflurane for the duration of the procedure.

Fetal position was mapped by detailed ultrasound scan (Corevision; Toshiba Medical Systems, Crawley, UK). Multiple port placement was performed for all prototypes. The first port was placed under ultrasound guidance; all subsequent ports were placed under direct vision to allow assessment of the individual system components. In all systems developed after method 1, the initial placement of a flexible guide wire (through an amnio needle) into the amniotic cavity under ultrasound guidance, was used to guide the placement of the various ports.

The InnerDyne Step access system designed for laparoscopic access (Autosuture; US Surgical, Norwalk, CT) was evaluated initially (method 1). This system comprises a radially expandable braided tubular sheath with a splittable cover, which protects the tubular braid during initial insertion. Within the sheath, a needle with a removable obturator can be placed to allow skin and uterine penetration. After the sheath has been advanced to its desired location, the needle is withdrawn. A rigid expansion port with obturator is introduced, thus expanding the braid sheath and splitting the protective covering. The obturator then can be removed leaving the fixed rigid port within the radially expanded sheath. Instruments then may be passed down through the valved expansion port.

The Veress needle used in this system for cutaneo-amniotic access did not provide a smooth transition to the sheath in this model. As a consequence, a rigid introducer was developed with a sharp bulbous end equal in diameter to the sheath, providing a ridge-free transition to the sheath (Fig 1; method 2). Unfortunately, removal of this introducer back through the expandable sheath caused splitting and fraying of the sheath distally and intussusception of the proximal end into the sheath handle. Addition of a mobile conical insert, which could be placed over the introducer into the sheath handle, resolved the sheath intussusception but did not alter the distal fraying (method 3). A slight improvement was achieved by the creation of a circumferential insert that could be passed down the radial expanding sheath, engaging the introducer and providing a tapered surface for the introducer to pull back through the distal sheath tip (Fig 2; method 4).

A rigid metallic port of equal diameter to the radial expanding port and solid introducer was investigated (method 5). The radially expanding port and handle were placed down the rigid lumen, which then was split along its axial length leaving the flexible sheath in place. This method overcame sheath fraying but negated any benefits of a flexible introduction system. This disadvantage was overcome by incorporating a peel-apart catheter introducer (Peel-apart Desilet; VYGON, Ecouen, France), which was placed percutaneously using the Seldinger technique (method 6). The radially expanding sheath then was place down the centre of the peel-apart sheath. The peel-apart catheter was split and removed as the expansion port with obturator was inserted. The potential for the peel-apart catheter introducer to act as a port was examined; however, there was continued amniotic fluid loss proximally because the introducer did not possess a valve.

The 10F Check-Flo Performer Introducer Set (Cook Incorporated, Bloomington, IN) incorporated a valve mechanism proximally allowing easy placement of instruments and a side channel, which could be used for irrigation (method 7). A 18FG spinal needle, preloaded with a superelastic shape-memory membrane tenting wire was inserted into the amniotic cavity next to the fetal head under ultrasound guidance. The shape-memory alloy wire was deployed and the needle withdrawn. The expanded wire was retracted gently, opposing the chorioamniotic membranes to the uterine wall (Fig 3). This stabilized an area of approximately $2 \mathrm{~cm}^{2}$ of amniotic membranes and allowed ultrasoundguided placement of a second spinal needle, through which a curved safe T-J double flexible wire guide (Cook Incorporated) was advanced into the amniotic cavity. The second needle was withdrawn and the cutaneoamniotic tract dilated with a 10F Check-Flo Performer Introducer Set.

This system was used to achieve tracheal occlusion as, on removal of the dilator, a rigid 3-mm $0^{\circ}$ laparoscope (Olympus Medical Systems, Keymed, Southend-on-Sea, England) was advanced into the fetal mouth and then into the trachea. The cutaneo-tracheal tract was secured by sliding the previously inserted $10 \mathrm{~F}$ port sheath until its rim was just visible past the telescope. The laparoscope then was replaced by a flexible choledochoscope (Olympus Medical Systems) incorporating an operating channel preloaded with a silicone balloon (Applied Medical Resources Corp, Medlink Europe, The Netherlands). The balloon was inflated $2 \mathrm{~cm}$ above the carina proximal to the right upper lobe bronchus. The instrument and port finally were withdrawn, the animals killed, and the cutaneo-tracheal tract assessed.

\section{RESULTS}

The 7 prototype systems were evaluated on 22 ewes with a total of 47 ports inserted. The first 6 systems all

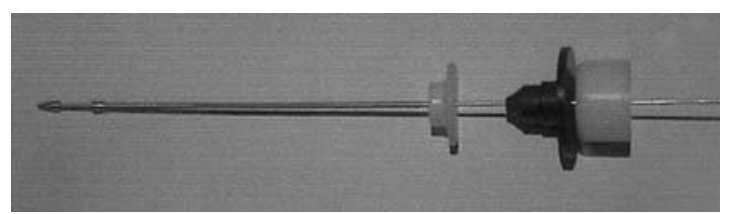

Fig 2. Evolution prototype of above to reduce incidence of fraying of distal tip and intussusception of proximal end of expandable port. 


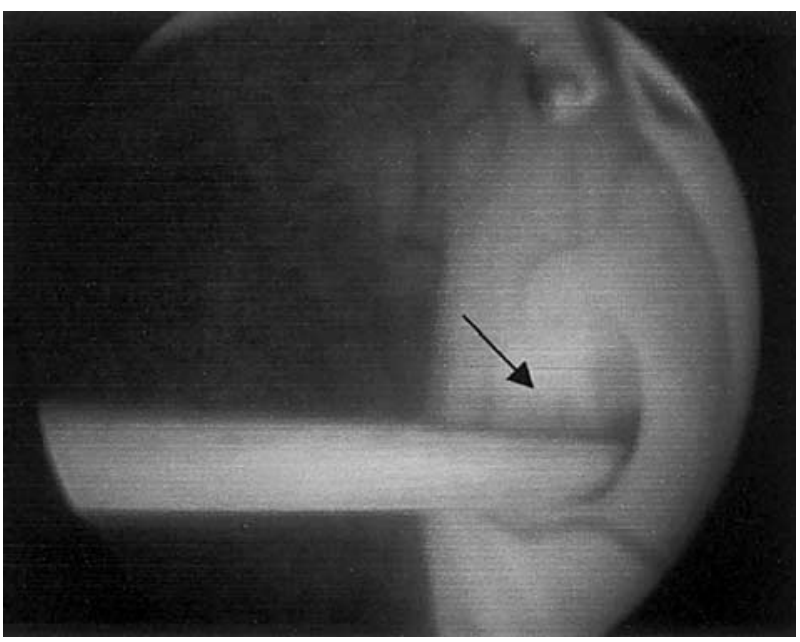

Fig 3. Membranes held by loop of superelastic memory shape alloy wire while instruments are passed into amniotic cavity. Note virtually no tenting of membranes at entry point (arrow).

were based on the radial expanding sheath, and subsequent stepwise modifications were designed to minimize membrane separation and tenting. The radially expanding sheath was limited to $15 \mathrm{~cm}$ in length, hence, with marked membrane tenting, the port was not of sufficient length to breach the membranes and enter the amniotic cavity. The alterations made to the introducer were partially successful in minimising the ridge between the radial expanding sheath and the cutting trochar, thereby reducing the possibility of membranes catching, and also reducing damage to the distal end of the sheath on removal. However, reliable percutaneous access could not be achieved with the use of the InnerDyne system, with occasional loss of amniotic cavity access owing to the port losing its anchored position. Furthermore, injury to some fetuses was noted, caused by the rigid introducer.

The use of the rigid split port did not overcome the problem of membrane tenting, but the rigid port was of sufficient length that the membranes could be allowed to tent until they were penetrated and slid up the side of the port. The port then was split and removed lengthwise. The necessity for an axial split was because of the radial expanding sheath handle onto which the valved rigid expansion port connects. The rigid obturator required by this system was associated with a high incidence of fetal injury owing to the depth and force required for placement.

The Seldinger approach utilised by the peel-apart catheter introducer required intra-amniotic placement of a needle and guide wire under real-time ultrasonography. This was associated with minimal tenting of the membrane, however, although finely tapered to the guide wire, the dilator with overlying sheath could, on occasion cause membrane tenting and failure of amniotic access of the sheath. There was no evidence of fetal trauma, because both the guide wire and dilator tips were flexible. The necessity for introduction of the InnerDyne port down the lumen of the split sheath was because of the lack of a valve on the split sheath, which was designed originally for removal after catheter placement.

The problem of membrane tenting was resolved with the development of a superelastic shape memory membrane tenting wire. This wire was deployed under ultrasound guidance through an amnio needle. The end of the wire assumed a preshape (figure of 8), which, when the wire was pulled back gently, held the uterine membranes against the uterine wall. This stabilised an area of $2 \mathrm{~cm}^{2}$ of amniotic membranes, through which placement of a 10F Check-Flo Performer Introducer Set was achieved (Fig 3). This system also overcame the problems of amniotic leakage because it incorporated a valve proximally. Furthermore, irrigation was feasible because of the presence of a side port. Percutaneous amniotic access, cannulation of the trachea, and intraluminal obstruction was achieved consistently in 6 cadaveric and 3 live anaesthetized ewes irrespective of fetal position (Figs 4 and 5). There was minimal leak around the catheter site, no trochar dislodgement, no extension tears or separation of chorioamniotic membranes at the points of entry, and no visible maternal or fetal trauma in any of the live experimental animals.

\section{DISCUSSION}

Minimally invasive systems rely on obtaining percutaneous access to an internal surgical site using smalldiameter trochars. The design of suitable trochars must fulfill many requirements, particularly those for fetoscopic access in a liquid-filled environment. All trochars should be able to be introduced with minimal trauma and risk of injury. The trochars used in fetoscopic access should be readily sealable to inhibit amniotic fluid leakage, and, in particular, should be designed to minimise leakage in the region surrounding the passage of the trochar through the chorioamniotic membranes and uterine wall. The expanded braid of the Innerdyne system provided both an anchoring and sealing function. The tubular braid shortens as it is expanded radially, causing the braid to tighten or clamp on the tissue with which it is in contact. Such tightening of the braid, when combined with the radial expansion, prevents separation of the tissue layers, providing excellent anchoring within the percutaneous penetration and enhances the peripheral seal within the uterine wall. A further advantage of the braided sheath was that it remained in place as the expansion port was withdrawn and replaced. Further anchoring may have been feasible either by including a Malecotlike structure or balloon at the distal end of the 

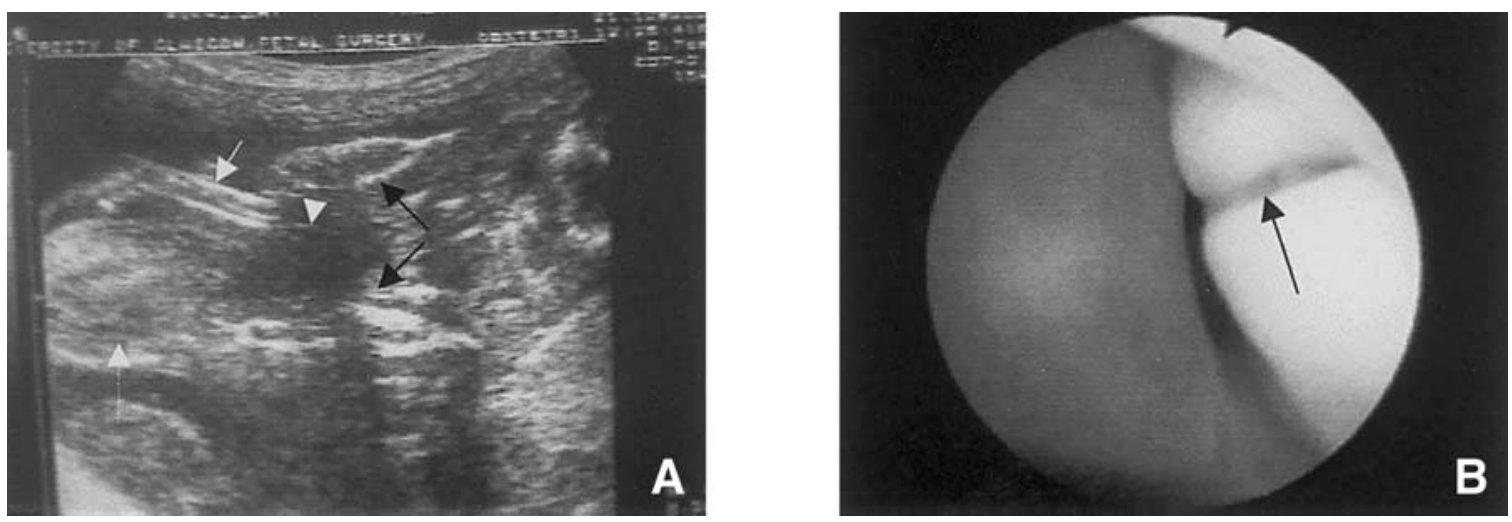

Fig 4. (A) Ultrasound image of port (white arrow) placement over guidewire (arrowhead) into fetal mouth (dashed arrow, snout; paired arrows, mandibular condyles). (B) Fetoscope approaching fetal mouth (arrow).

expansion port, which would ensure that the distal end of the port remained within the amniotic cavity after deployment. ${ }^{7}$ An external clamp at the proximal end of the expansion port so that the abdominal and uterine wall disposed between the anchors may be captured would further enhance the anchoring potential of the port.

The plurality afforded by the radially expanding braid for different diameter expansion ports was not required. Although capable of expanding from less than $3 \mathrm{~mm}$ up to 5 or $15 \mathrm{~mm}$, minimisation of risk of preterm premature rupture of membranes dictates that the access hole be of a minimum diameter and all instruments be miniaturised appropriately. ${ }^{8}$ Therefore, although the radially expanding dilator system is advantageous in that it reduces the initial trauma and facilitates subsequent exchange of different-sized cannulae with radial collapse of the conformable sheath, we do not envisage that this will be required for most fetal surgical applications that use instruments less than $3 \mathrm{~mm}$ in diameter. Future applications may be hindered by rigidity of the expansion port access lumen, which cannot accommodate instruments and articles with irregular shapes. The use of the percu- taneous radially expanding sheath as the port would overcome this disadvantage and allow novel instruments to reach the amniotic cavity.

The similarities of the principles required for access to vasculature and amniotic cavity access are striking. The Seldinger technique requires initial access with a needle, passage of a guide wire, and then radial dilation of the tract to accommodate a larger introducer sheath. It is not clear, however, whether the probability of prelabour premature rupture of membranes (PPROM) associated with this Seldinger method of access would be the risk owing to size of the needle trochar primary defect, ie, $2 \%,{ }^{8}$ or the risk attributable to the final port size, which has been noted to be as high as $20 \% .^{9}$ We did not continue these animals to term because our primary objective was to show the feasibility of percutaneous tracheal occlusion at 0.75 gestation, a time-point that would correspond to 30 weeks in humans. We, therefore, were unable to determine the risk of PPROM attributable to the fetoscopic port. To date, the closure of fetoscopic sites has remained elusive, although recent studies have shown that a combination of liquid polyethylene glycol

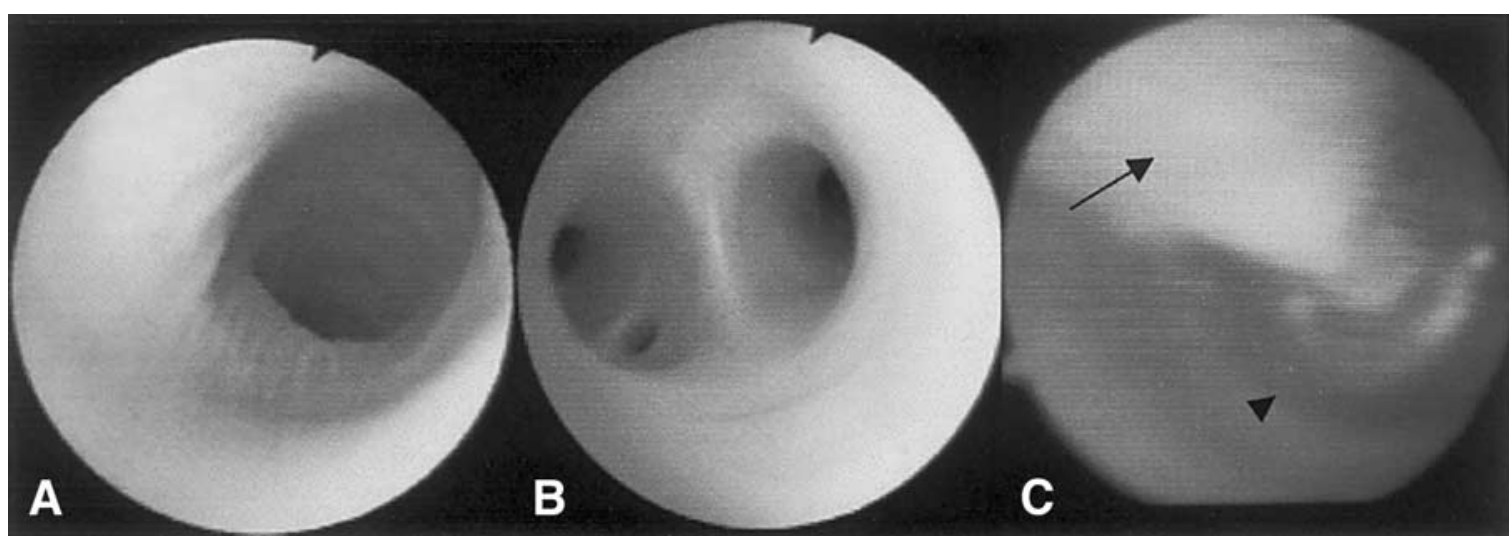

Fig 5. (A) View of the fetal trachea. Note tracheal rings superolaterally. (B) Fetoscope passed to carina. (C) Balloon (arrowhead) deployed 2 cm proximal to carina (balloon shaft marked by arrow). 
and microfibrillar collagen may enable efficient sealing of membrane defects as assessed both in vitro and in vivo. ${ }^{10}$

The implementation of the superelastic shape memory alloy was required because of the unique anatomy of the ovine uterus with its loosely adherent chorioamniotic membranes. Although these could have been applied to the original radial expanding system, they would not have prevented the other complications of fetal injury and trochar dislodgement, which would require flexible introducers and longer sheaths-inherent properties of vascular catheters.

To date, both the radially expanding sheath system ${ }^{2}$ and the Check-Flo Performer Introducer Set have been used successfully for uterine access in humans. A single 5-mm transmyometrial radially expanding sheath port was placed postlaparotomy to facilitate endoluminal tracheal occlusion; however, both cases were complicated by PPROM, which may be related to port size and possible chorioamniotic membrane separation. We have used a percutaneously placed Check-Flo system without the necessity for the superelastic shape memory alloys to allow cord occlusion for isolated fetal anomaly in twins with no incidence of PPROM.

Fetal tracheal occlusion induces lung growth in experimentally ${ }^{12-15}$ and clinically induced pulmonary hypopla- sia. ${ }^{2,11}$ A variety of techniques have been used to temporarily and reversibly occlude the trachea, including initially external clips. ${ }^{16}$ Subsequent research has focused on intraluminal occlusion devices: sponges, ${ }^{17} \mathrm{um}$ brellas, ${ }^{18}$ and detachable balloons, ${ }^{19,20}$ and the delivery of these devices by minimally invasive techniques. To date, all these procedures have required maternal laparotomy. The percutaneous method described appears to provide reliable fetoscopic access in the ovine model. The amniotic cavity is first accessed by a fine needle to allow placement of a memory-shape alloy wire, which minimizes membrane tenting and separation, subsequent to the insertion of a fetoscopic port. However, discrepancies in anatomy make the technical approach to fetoscopy simpler in humans, and we have shown clinically that reliable percutaneous access with minimal membrane separation in patients can be achieved using the described port and Seldinger technique alone.

Minimally traumatic percutaneous access now is achievable both in the ovine model and in humans; future research should concentrate on the postprocedure closure of chorioamniotic membranes to further reduce the risk of fetoscopic procedures.

\section{ACKNOWLEDGMENTS}

The authors thank Dr Michael Wilkinson and Ms Christine Stirton and her staff for veterinary care and technical assistance.

\section{REFERENCES}

1. Luks FI, Deprest JA: Endoscopic fetal surgery: A new alternative?. Eur J Obstet Gynecol Reprod Biol 52:1-3, 1993

2. Harrison MR, Albanese CT, Hawgood SB, et al: Fetoscopic temporary tracheal occlusion by means of detachable balloon for congenital diaphragmatic hernia. Am J Obstet Gynecol 185:730-733, 2001

3. Bhoyrul S, Mori T, Way LW: Radially expanding dilatation. A superior method of laparoscopic trocar access. Surg Endosc 10:775778, 1996

4. Dreyfus M, Becmeur F, Schwaab C, et al: The pregnant ewe: An animal model for fetoscopic surgery. Eur J Obstet Gynecol Reprod Biol 71:91-94, 1997

5. Luks FI, Deprest JA, Vandenberghe K, et al: Fetoscopy-guided fetal endoscopy in a sheep model. J Am Coll Surg 178:609-612, 1994

6. Wintour EM, Laurence BM, Lingwood BE: Anatomy, physiology and pathology of the amniotic and allantoic compartments in the sheep and cow. Aust Vet J 63:216-221, 1986

7. Kohl T, Szabo Z, Suda K, et al: Percutaneous fetal access and uterine closure for fetoscopic surgery. Lessons learned from 16 consecutive procedures in pregnant sheep. Surg Endosc 11:819-824, 1997

8. Devlieger R, Gratacos E, Ardon H, et al: Factors influencing the flow rate through a surgical defect in human fetal membranes. Prenat Diagn 22:201-205, 2002

9. Gratacos E, Deprest J: Current experience with fetoscopy and the Eurofoetus registry for fetoscopic procedures. Eur J Obstet Gynecol Reprod Biol 92:151-159, 2000

10. Devlieger R, Brandenburh H, Verbist L, et al: Sealing fetal membrane defect using microfibrillar collagen, liquid polyethylene glycol (PEG) or a mixture of both: evaluation in vitro in organ cultures and in vivo in the rabbit. J So Gynecol Invest 9:222A-222A, 2002

11. Flake AW, Crombleholme TM, Johnson MP, et al: Treatment of severe congenital diaphragmatic hernia by fetal tracheal occlusion Clinical experience with fifteen cases. Am J Obstet Gynecol 183:10591066, 2000

12. Hedrick MH, Estes JM, Sullivan KM, et al: Plug the lung until it grows (PLUG): A new method to treat congenital diaphragmatic hernia in utero. J Pediatr Surg 29:612-617, 1994

13. Kitano Y, Kanai M, Davies P, et al: BAPS prize-1999: Lung growth induced by prenatal tracheal occlusion and its modifying factors: A study in the rat model of congenital diaphragmatic hernia. J Pediatr Surg 36:251-259, 2001

14. Nardo L, Hooper SB, Harding R: Lung hypoplasia can be reversed by short-term obstruction of the trachea in fetal sheep. Pediatr Res 38:690-696, 1995

15. Benachi A, Chailley-Heu B, Delezoide AL, et al: Lung growth and maturation after tracheal occlusion in diaphragmatic hernia. Am J Respir Crit Care Med 157:921-927, 1998

16. VanderWall KJ, Bruch SW, Meuli M, et al: Fetal endoscopic ('Fetendo') tracheal clip. J Pediatr Surg 31:1101-1103, 1996

17. Bealer JF, Skarsgard ED, Hedrick MH, et al: The 'PLUG' odyssey: Adventures in experimental fetal tracheal occlusion. J Pediatr Surg 30:361-364, 1995

18. Luks FI, Gilchrist BF, Jackson BT, et al: Endoscopic tracheal obstruction with an expanding device in a fetal lamb model: Preliminary considerations. Fetal Diagn Ther 11:67-71, 1996

19. Benachi A, Dommergues M, Delezoide AL, et al: Tracheal obstruction in experimental diaphragmatic hernia: An endoscopic approach in the fetal lamb. Prenat Diagn 17:629-634, 1997

20. Chiba T, Albanese CT, Farmer DL, et al: Balloon tracheal occlusion for congenital diaphragmatic hernia: Experimental studies. J Pediatr Surg 35:1566-1570, 2000 


\section{Discussion}

J. Langer (Toronto, Ontario): The technique of single port balloon placement already has been used for a number of years in San Francisco.

C.A. Hajivassilou (response): I am aware of this, but I have not seen any published report of this being used totally percutaneously without a laparotomy being used to deliver part of the uterus.

J. Langer (Toronto, Ontario): Fixation of the membranes is a very nice technique for keeping the membranes up against the uterine wall during access to the uterus, but the big problem in these patients is after you have taken everything out; many of these patients will have amniotic fluid leakage because of a failure to seal the membranes afterwards. Do you have any ideas for that?

C.A. Hajivassilou (response): This has been described as the Achilles heel of fetoscopic interventions. We have developed a blueprint of a double lumened cannula that will be inserted through the port during exit of the port, and through that cannula we will be deploying a 2-component glue substance that will activate itself on contact with the amniotic fluid and seal the membrane.

M.P. Kings (London, UK England): This is a comment rather than a question. We have undertaken this technique in humans and now have operated on 5 patients, and 2 further patients are planned this week. We have not found free-floating membranes to be a problem in the human patient. We are very fortunate in having a very skilled fetoscopist in Nicolaides and in collaboration with Jan de Prest from Belgium who developed the single port system. In terms of the question about leakage of amniotic fluid, Jan de Prest has just published a paper again in the ovine model on fibrin plugging of any amniotic seals.

C.A. Hajivassilou (response): The membranes are not a problem in the humans. All this development has been really a step to develop the technique to allow fetoscopic research in animals, and we had to solve the problem of the floating membranes, which is a specific problem in the ovine model. The uterine access in the human is exceedingly easy if you compare it with trying to achieve access in the ovine model. 